\title{
EXISTENCE THEORY FOR GAMES OF PRICING AND TECHNOLOGY
}

\author{
KOKOU Y. ABALO ${ }^{1}$ and MICHAEL M. KOSTREVA ${ }^{2}$
}

(Received 15 March, 1999; revised 19 January, 2000)

\begin{abstract}
A differential game model of a technological service industry is reformulated as an equivalent game over a function space by direct substitution of the solutions of the state equations. For this game, Nash equilibria are shown to exist under certain mild assumptions. A generalization is considered in which each firm has a choice of three different objective functions, which may refiect distinct management options in a technological service industry. Nash equilibria for the generalized version exist under similar mild assumptions.
\end{abstract}

\section{Introduction}

In modern economic environments, the service industries have advanced to occupy a key position. With rapidly advancing technologies, other related industries increase their influence. The study of Flexible Manufacturing Systems (FMS) has become more and more important due to the advance of technology. The investment in FMS is analyzed through a game theoretical model in Tombak [10]. In Cheng and Dinopoulos [2] the relevance of the introduction of new products and new processes to global business fluctuations is shown as an implication of technological changes. While the ideas in these two papers are developed in the light of the Schumpeterian line of thought, Roller and Tombak [9] use a 2-stage game to predict the correlation between the size of a market, the level of differentiation of products and the proportion of FMS firms being sustained. With the application of advanced technology there will emerge a manufacturing sector with little or no inventory, and a structure very similar to a service industry. Hence, the mathematical models of such industries will increase in importance, and it becomes crucial to understand, in more mathematical detail, their foundations.

\footnotetext{
'Department of Mathematics, Erskine College, Due West, SC 29639, USA.

${ }^{2}$ Department of Mathematical Sciences, Clemson University, Clemson, SC 29634-0975, USA.

(C) Australian Mathematical Society 2002, Serial-fee code 1446-1811/02
} 
In an earlier work, Kostreva [5] formulated an $N$-firm differential game model that modeled the application of new technology in a service industry. It captured the profit and the capacity management features of each firm in one objective functional. By applying necessary optimality conditions, and through numerical solutions of those conditions, a detailed case and scenario study was presented. The purpose of the current paper is to present detailed existence proofs for the Nash equilibrium of the same model, under very mild assumptions, and to show that some natural generalizations also have solutions. The differential game studied earlier included the following model for player $i$ :

$$
\begin{gathered}
\text { Maximize } \int_{0}^{T}\left\{\left[p_{i}(t)-b_{i}(t)-B_{i}(t)\right] D_{i}(t)-m_{i}(t) q_{i}(t)-c_{i}(t) a_{i}(t)+s_{i}(t) r_{i}(t)\right. \\
\left.-w_{i}\left[\partial_{i} q_{i}(t)-D_{i}(t)\right]^{2}\right\} E_{i}(t) d t+h_{i} q_{i}(T) E_{i}(T)
\end{gathered}
$$

subject to

$$
\begin{gathered}
q_{i}^{\prime}(t)=a_{i}(t)-r_{i}(t), \quad q_{i}(0)=q_{i 0}, \\
b_{i}^{\prime}(t)=-\alpha_{i}(t) a_{i}(t) b_{i}(t), \quad b_{i}(0)=b_{i 0}, \\
0 \leq q_{i}(t)-r_{i}(t), \\
0 \leq p_{i}(t) \leq P_{i}(t), \\
0 \leq a_{i}(t) \leq A_{i}(t), \\
0 \leq r_{i}(t) \leq R_{i}(t),
\end{gathered}
$$

for all $t \in[0, T]$, where

$$
\begin{gathered}
D_{i}(t)=d_{i 0}(t)-d_{i i} p_{i}(t)+\sum_{j \neq i} d_{i} p_{j}(t), \quad E_{i}(t)=e^{-\rho_{i} t}, \\
0 \leq \partial_{i} \leq 1, \quad w_{i} \geq 0, \quad \alpha_{i}(t) \geq 0
\end{gathered}
$$

for all $t \in[0, T]$.

In order to completely specify the model, the following description of the variables and inputs is given:

$t$

$T$

$i$

$q_{i}(t) \quad$ level of productive capacity

$b_{i}(t) \quad$ level of per unit production cost which is reducible through the acquisition of new technology

$q_{i}$ and $b_{i}$ state variables of firm $i$

$a_{i}(t) \quad$ rate of acquisition of new technology 


\begin{tabular}{|c|c|}
\hline$r_{i}(t)$ & rate of retirement of existing capacity \\
\hline$p_{i}(t)$ & price of the product of firm $i$ \\
\hline$a_{i}, r_{i}$ and $p_{i}$ & control variables of firm $i$ \\
\hline$D_{i}(t)$ & level of demand of $i^{\text {th }}$ firm's product \\
\hline$d_{i 0}(t)$ & base level of demand for firm $i$ \\
\hline$d_{i i}, d_{i j}, j \neq i$ & input constants for demand equations \\
\hline$B_{i}(t)$ & $\begin{array}{l}\text { any component of cost for firm } i \text { that is unaffected by the acqui- } \\
\text { sition of a new technology }\end{array}$ \\
\hline$m_{i}(t)$ & maintenance costs of the $i^{\text {th }}$ firm \\
\hline$h_{i}$ & per unit firm $i$ capacity salvage value at time $T$ \\
\hline$c_{i}(t)$ & $\begin{array}{l}\text { per unit cost for firm } I \text { of acquiring and implementing new tech- } \\
\text { nology }\end{array}$ \\
\hline$s_{i}(t)$ & $\begin{array}{l}\text { per unit firm } i \text { revenue received (or the costs incurred) due to } \\
\text { retirement of existing capacity at time } t\end{array}$ \\
\hline$\rho_{i}$ & continuous discount rate \\
\hline$E_{i}(t)$ & discounting experienced at time $i$ \\
\hline$\partial_{i}$ & $\begin{array}{l}\text { target utilization coefficient of productive capacity, set by man- } \\
\text { agement of firm } i\end{array}$ \\
\hline$w_{i}$ & $\begin{array}{l}\text { weight (penalty cost) per unit squared deviation between the ac- } \\
\text { tual level of demand and the target level of capacity utilization }\end{array}$ \\
\hline$\alpha_{i}(t)$ & $\begin{array}{l}\text { effectiveness of a unit acquisition of firm } i \text { technology in reducing } \\
\text { the per unit production cost at time } t \text {. }\end{array}$ \\
\hline
\end{tabular}

REMARKS. The above game has several features which place it outside the realm of "standard" differential game theory (see, for example, Friedman [3]). First, the objective functional, although quadratic, involves some nonlinear terms which multiply state variables by control variables. In the "standard" differential game with quadratic objective, these nonlinearities are not present in the quadratic functionals. Second, the state equation (1.3), which describes the technological cost function, is nonlinear, appearing as a product of a state variable and a control variable. Finally, there is the state constraint (1.4) which is necessary, but complicates matters. The coupling constraints on demand (1.8) play a significant role in the model, and the coefficients must be carefully obtained by econometric analysis. However, for all ranges of realistic data, it is not necessary to restrict demand to be nonnegative. If any company has demand equal to zero, it would signal the end of that phase of competition and the model would not apply. Such a constraint is redundant in the present context. Hence it may be omitted for the purposes of the current paper.

In the next section it will be shown how to transform the differential game (1.1)(1.8) into an infinite game without state equations. Such a transformation has not been applied before to this game or any of its variants. The insights gained by 
making the transformation are key to understanding how to guarantee the existence of Nash solutions. The techniques we use are similar to those proposed in Varaiya [12] and Vidyasagar [13], but they are different because these papers consider only linear differential equations, while our model considers some nonlinear, integrable state equations.

\section{Reformulation}

Assuming that $P_{i}, A_{i}, R_{i}$ are bounded piecewise continuous functions on $[0, T]$ the solution to the differential system (1.2)-(1.6) is given by

$$
\begin{aligned}
& q_{i}(t)=q_{i 0}+\int_{0}^{t}\left(a_{i}(\tau)-r_{i}(\tau)\right) d \tau, \\
& b_{i}(t)=b_{i 0} \exp \left(-\int_{0}^{t} \alpha_{i}(\tau) a_{i}(\tau) d \tau\right),
\end{aligned}
$$

for $r_{i}(t) \in\left[0, S_{i}(t)\right]$, where

$$
S_{i}(t)=\min \left\{R_{i}(t), q_{i 0}+\int_{0}^{t} A_{i}(\tau) d \tau\right\}, \quad t \in[0, T] .
$$

Here $S_{i}$ is a bounded piecewise continuous function on $[0, T]$ since it is the minimum of bounded piecewise continuous functions. By (2.3), the inequalities (1.4) and (1.7) are automatically enforced. Now, by substituting (2.1) and (2.2) into (1.1) and (1.4)-(1.7) the original problem (1.1)-(1.6) becomes

$$
\begin{aligned}
\operatorname{Max} \int_{0}^{T}\left\{\left[p_{i}(t)-b_{i 0} e^{-\int_{0}^{\prime} \alpha_{i}(\tau) a_{i}(\tau) d \tau}-B_{i}(t)\right]\left[d_{i 0}(t)-d_{i i} p_{i}(t)+\sum_{j \neq i} d_{i j} p_{j}(t)\right]\right. \\
-m_{i}(t)\left[q_{i 0}+\int_{0}^{t}\left(a_{i}(\tau)-r_{i}(\tau)\right) d \tau\right]-c_{i}(t) a_{i}(t)+s_{i}(t) r_{i}(t) \\
-w_{i}\left[\partial_{i}\left(q_{i 0}+\int_{0}^{t}\left(a_{i}(\tau)-r_{i}(\tau)\right) d \tau\right)\right. \\
\left.\left.\quad-\left(d_{i 0}(t)-d_{i i} p_{i}(t)+\sum_{j \neq i} d_{i j} p_{j}(t)\right)\right]^{2}\right\} E_{i}(t) d t \\
+h_{i} \int_{0}^{T}\left(a_{i}(\tau)-r_{i}(\tau)\right) d \tau E_{i}(T)
\end{aligned}
$$

subject to

$$
0 \leq a_{i}(t) \leq A_{i}(t), \quad 0 \leq p_{i}(t) \leq P_{i}(t), \quad 0 \leq r_{i}(t) \leq S_{i}(t),
$$

for all $t \in[0, T]$. 
Since $A_{i}, P_{i}$ and $R_{i}$ are assumed to be bounded piecewise continuous functions on $[0, T]$, the functions $A_{i}, P_{i}, S_{i}$ may be bounded on $[0, T]$ respectively by say $M_{i 1}$, $M_{i 2}$ and $M_{i 3}$. These assumptions are justified, since the rate of acquisition of new technology, the rate of retirement of existing capacity and the price of the product must be bounded over $[0, T]$.

Even though the game and (2.4)-(2.5) are equivalent, the infinite programming game presents some advantages. First, there are no state equations involved in the constraints in the form of differential systems. Second, the constraints (2.5) have an advantageous mathematical structure that will be discussed in the next section. Third, the functional (2.4) is easier to understand as far as its continuity and its concavity in the players' strategies are concerned.

Later the functional (2.4) will be regarded as the nonnegative linear combination of two functionals. This will enable us to analyze several variants of the initial game (2.4)-(2.5).

It should be observed that the above differential game is the "natural" formulation. It was derived specifically for a technologically driven service industry in Kostreva [5] as a game theoretic generalization of the model in Gaimon [4]. At the same time, the new formulation, as a game on an infinite dimensional space, is more tractable mathematically. It would not be a formulation which would occur "naturally", but it is valuable as a mathematical analysis structure. It seems that there is, in general, a pair of game formulations, one differential, one non-differential, over an infinite dimensional space. This pair of formulations will always have certain characteristics as described above for the game of this paper. Each member of the pair has value, and is useful in its own context.

Observe that the functional $J_{i}$ is a nonnegative linear combination of two functionals $J_{i 1}$ and $J_{i 2}$, where

$$
\begin{aligned}
J_{i 1}(u)= & \int_{0}^{T}\left\{\left[p_{i}(t)-b_{i 0} e^{-\int_{0}^{\prime} \alpha_{i}(\tau) a_{i}(\tau) d \tau}-B_{i}(t)\right]\right. \\
& \times\left[d_{i 0}(t)-d_{i i} p_{i}(t)+\sum_{j \neq i} d_{i j} p_{j}(t)\right]-m_{i}(t)\left[q_{i 0}+\int_{0}^{t}\left(a_{i}(\tau)-r_{i}(\tau)\right) d \tau\right] \\
& \left.-c_{i}(t) a_{i}(t)+s_{i}(t) r_{i}(t)\right\} E_{i}(t) d t+h_{i} \int_{0}^{T}\left(a_{i}(\tau)-r_{i}(\tau)\right) d \tau E_{i}(T)
\end{aligned}
$$

and

$$
\begin{aligned}
J_{i 2}(u)= & \int_{0}^{T}\left[\partial_{i}\left(q_{i 0}+\int_{0}^{t}\left(a_{i}(\tau)-r_{i}(\tau)\right) d \tau\right)\right. \\
& \left.-d_{i 0}(t)-d_{i i} p_{i}(t)+\sum_{j \neq i} d_{i j} p_{j}(t)\right]^{2} E_{i}(t) d t .
\end{aligned}
$$


Writing the functional $J_{i}$ as $J_{i 1}+w_{i} J_{i 2}$ allows the following interpretation: $J_{i 1}$ represents profit while $J_{i 2}$ represents capacity management.

\section{Notation and theory}

Let $I=\{1, \ldots, N\}$ be the set of players and for each $i \in I$, denote by $\Omega_{i}$ the nonempty set of the $i^{\text {th }}$ player's strategies. Assume that $\Omega_{i} \subset L_{3}^{2}[0, T]$, the square integrable triples of functions on [0,T]. Elements of $\Omega_{i}$ will be denoted by $u_{i}$. Let $J_{i}$ denote the $i^{\text {th }}$ player's payoff functional defined on $\Omega=\prod_{i=1}^{N} \Omega_{i}$.

The following notation will also be used:

$$
-i=I-\{i\}, \quad u_{-i}(\cdot)=\prod_{k \in-i} u_{k}(\cdot), \quad \Omega_{-i}=\prod_{k \in-i} \Omega_{k}, \quad u_{i}(u)=\arg \max _{u_{i} \in \Omega_{j}} J_{i}\left(u_{i}, \cdot\right) .
$$

Finally, the game will be denoted by $\Gamma=\left(I, J_{i}, \Omega_{i}\right)$ and throughout this paper the following two well-known results will be used.

THEOREM (Tychonoff [11]). Let $K$ be a nonempty compact convex set in a locally convex topological Hausdorff vector space $X$. Then any continuous function from $K$ into $K$ has a fixed point.

THEOREM (Polyak [7]). A functional attains a unique maximum on every closed bounded convex set of a reflexive space if and only if it is strictly quasi-concave.

For the purpose of this specific problem the above theorem of Polyak can be stated in the following equivalent way.

THEOREM. A functional attains a unique maximum on every convex weakly compact set of a reflexive space if and only if it is strictly quasi-concave.

The structure of the players' strategy sets is given by the following theorem.

THEOREM. For each $i \in I$, the set $\Omega_{i}$ is convex and weakly compact.

ProOF. First we will show that the set $\left\{a_{i}(t), a_{i}(t) \in\left[0, A_{i}(t)\right], t \in[0, T]\right\}$ is convex and weakly compact and then complete the proof by noticing that $\Omega_{i}$ is the Cartesian product of convex and weakly compact sets.

The set $\left\{a_{i}(t), a_{i}(t) \in\left[0, A_{i}(t)\right], t \in[0, T]\right\}$ is obviously convex; it is also bounded since $A_{i}$ is bounded piecewise continuous on $[0, T]$.

By a result of Levinson [6], if a sequence of feasible strategies $\left\{a_{n}(t)\right\}_{n \geq 1}$ converges weakly to $a_{0}(t)$, then except on sets of measure zero, $a_{0}(t) \in\left[0, A_{i}(t)\right]$. This shows that $\left[0, A_{i}(t)\right]$ is closed in $L^{2}[0, T]$. 
Also, each feasible sequence has a convergent subsequence (see [8]). Therefore we conclude that $\left[0, A_{i}(t)\right]$ is weakly compact in $L^{2}[0, T]$. And thus $\Omega_{i}$ is weakly compact in $L_{3}^{2}[0, T]$ as the Cartesian product of weakly compact sets.

In the next section, the Profit Game and then the Capacity Management Game will be studied. Following these two results, the existence of a Nash equilibrium of the Change of Technology Game and of a related family of Games will be addressed.

\section{Main results}

\subsection{The Profit Game}

THEOREM. The Profit Game, that is, $\Gamma^{1}=\left(I, J_{i 1}, \Omega_{i}\right)$, has a Nash equilibrium point if for each $i \in I, b_{i 0} \geq 0$ and $d_{i i}>0$.

PROOF. Denote by $g_{i 1}(\cdot)$ and $g_{i 2}(\cdot)$ the mappings from $\Omega$ into itself defined by:

$$
\begin{aligned}
g_{i 1}= & -b_{i 0} \exp \left(-\int_{0}^{t} \alpha_{i}(\tau) a_{i}(\tau) d \tau\right) \\
g_{i 2}= & p_{i}(t)\left(d_{i 0}(t)-d_{i i} p_{i}(t)+\sum_{j \neq i} d_{i j} p_{j}(t)\right) \\
& -m_{i}(t)\left(q_{i 0}-\int_{0}^{t}\left(a_{i}(\tau)-r_{i}(\tau)\right) d \tau\right)-c_{i}(t) a_{i}(t)+s_{i}(t) r_{i}(t) E_{i} \\
& +h_{i}\left(q_{i 0}-\int_{0}^{T}\left(a_{i}(\tau)-r_{i}(\tau)\right) d \tau\right) E_{i}(T) .
\end{aligned}
$$

Since $b_{i 0} \geq 0$, the functional $g_{i 1}(u)$ is concave in $a_{i}(t)$. On the other hand, the functional $g_{i 2}(u)$ is linear in $a_{i}(t), r_{i}(t)$ and quadratic in $p_{i}(t)$. It is strictly concave in $p_{i}(t)$ for $d_{i i}>0$. Therefore $J_{i 1}(u)$ will be strictly concave in $u_{i}(t)$ if $b_{i 0} \geq 0$ or $d_{i i}>0$ as the sum of strictly concave functions. Also $J_{i 1}\left(u_{i}, \cdot\right)$ is clearly continuous on $\Omega$.

Let $\Omega_{i} \subset\left(L_{3}^{2}[0, T], \tau_{i}\right)$, where $\tau_{i}$ is the weak topology of $L_{3}^{2}[0, T]$ and $\tau=$ $\prod_{i=1}^{N} \tau_{i}$. Then the set $(\Omega, \tau)$ is compact in $\left(\prod_{i=1}^{N} L_{3}^{2}[0, \tau], \tau\right)$. By the result of Polyak mentioned above, the set $u_{i 1}(u)=\arg \max _{u_{i} \in \Omega_{i}} J_{i 1}\left(u_{i}, \cdot\right)$ is a singleton and thus the multifunction $u_{i 1}(u)$ is continuous on $\left(\Omega_{i}, \tau_{i}\right)$.

Consider the mapping $u(\cdot):(\Omega, \tau) \mapsto(\Omega, \tau)$

$$
u \mapsto\left(u_{11}(u), \ldots, u_{N 1}(u)\right)
$$

which is a continuous mapping as the composition and the Cartesian product of continuous mappings. The Profit Game has a Nash equilibrium $u^{*}$ if and only if $u^{*}$ is 
a fixed point of the mapping $u(\cdot)$. Since $(\Omega, \tau)$ is a convex and compact set, it follows from Tychonoff's fixed point theorem that $u(\cdot)$ has a fixed point $u^{*}$. This concludes the proof of the existence of a Nash point.

The theorem above guarantees the existence of a Nash equilibrium for the Profit Game as long as each firm has a nonnegative initial level of per unit production cost which is reducible through the acquisition of new technology along with a positive constant input $d_{i i}$. Under these assumptions, the price of the products can be lowered and the demand equation becomes a strictly decreasing function of the price component of each firm's strategy. In summary, the demand increases as the price of the product decreases, which agrees with classical economic theory.

\subsection{The Capacity Management Game}

THEOREM. If for each $i \in I, d_{i i}>0$ and $\partial_{i} \neq 0$, then the Capacity Management Game $\Gamma^{2}=\left(I, J_{i 2}, \Omega_{i}\right)$ has a Nash equilibrium point.

PROOF. The functional $J_{i 2}\left(u_{i}, \cdot\right)$ is quadratic in $u_{i}$ and strictly concave in $u_{i}$, if $\partial_{i} \neq 0$ and $d_{i i}>0$. Also $J_{i 2}\left(u_{i}, \cdot\right)$ is clearly continuous on $\Omega_{i}$. Therefore the set defined by $u_{i 2}(u)=\arg \max _{u_{i} \in \Omega_{i}} J_{i 2}\left(u_{i}, \cdot\right)$ is a singleton and thus the multifunction $u_{i 2}(u)$ is continuous on $\left(\Omega_{i}, \tau_{i}\right)$.

Consider the mapping $u(\cdot):(\Omega, \tau) \mapsto(\Omega, \tau)$

$$
u \mapsto\left(u_{12}(u), \ldots, u_{N 2}(u)\right)
$$

which is a continuous mapping as the composition and the Cartesian product of continuous mappings. The Capacity Management Game has a Nash equilibrium $u^{*}$ if and only if $u^{*}$ is a fixed point of the mapping $u(\cdot)$. Since $(\Omega, \tau)$ is a convex and compact set, it follows from Tychonoff's fixed point theorem that $u(\cdot)$ has a fixed point $u^{*}$ and such a point is Nash. The proof is complete.

The Nash equilibrium of $\Gamma^{2}$ is shown to exist under very weak conditions. As a matter of fact, in an industrial environment, the target utilization coefficient of productive capacity, that is, $\partial_{i}$ in this context, cannot objectively be equal to zero unless bankruptcy is a desirable option for the industrial unit. In this game, the acquisition of a new technology must increase the capacity of production. Another impact the acquisition of new technology must have on the production is the increase in demand as the price of the product decreases. In other words the demand equation must be a strictly decreasing function in the price component of each firm's strategy. This is made possible whenever the input constant $d_{i i}$ is positive for each $i$.

The following paragraph addresses the initial problem (2.4)-(2.5). The existence of a Nash equilibrium of the Change of Technology Game comes as a consequence of the two existence theorems proved above. 


\subsection{The Change of Technology Game}

THEOREM. If for each $i \in I, d_{i i}>0$ and either $b_{i 0} \geq 0$ or $\partial_{i} \neq 0$, then the Change of Technology Game, that is, $\Gamma=\left(I, J_{i 1}+w_{i} J_{i 2}, \Omega_{i}\right)$, has a Nash equilibrium point.

PROOF. Here $J_{i 1}\left(u_{i}, \cdot\right)+w_{i} J_{i 2}\left(u_{i}, \cdot\right)$ is strictly concave and continuous on $\left(\Omega_{i}, \tau_{i}\right)$ as the nonnegative linear combination of strictly concave and continuous functions. By Polyak [7] the set $u_{i}(u)=\arg \max _{u_{i} \in \Omega_{i}}\left(J_{i 1}\left(u_{i}, \cdot\right)+w_{i} J_{i 2}\left(u_{i}, \cdot\right)\right)$ is a singleton and thus the multifunction $u(u)$ is continuous on $\left(\Omega_{i}, \tau_{i}\right)$.

Consider the mapping $u(\cdot):(\Omega, \tau) \mapsto(\Omega, \tau)$

$$
u \mapsto\left(u_{1}(u), \ldots, u_{N}(u)\right)
$$

which is a continuous mapping as the composition and the Cartesian product of continuous mappings. The Change of Technology Game has a Nash equilibrium $u^{*}$ if and only if $u^{*}$ is a fixed point of the mapping $u(\cdot)$. Since $(\Omega, \tau)$ is a convex and compact set, it follows from Tychonoff's fixed point theorem that $u(\cdot)$ has a fixed point $u^{*}$. This concludes the proof of the existence of a Nash point.

In the comments following the Profit and the Capacity Management Games results we have mentioned the weakness of all the assumptions stated in the theorem above. However, in this more general game, the conditions for the existence of a Nash equilibrium are even weaker. In fact, the nonnegativity of the per unit production cost which is reducible through the acquisition of new technology and the non zero value of the target utilization coefficient of production capacity of each firm play an equivalent role in the existence of a Nash equilibrium of the Change of Technology Game. This gives the game flexibility since these two conditions are not mutually exclusive.

The following paragraph takes advantage of breaking the initial game into several other games with a different interpretation for each game. The theorem that will be proved offers three different options to each firm to compete in the game without jeopardizing the existence of a Nash equilibrium.

\subsection{Related Games and Extensions}

THEOREM. Let $F_{i} \in\left\{J_{i}, J_{i 1}, J_{i 2}\right\}$. If $b_{i 0} \geq 0, d_{i i}>0$ and $\partial_{i} \neq 0$, for each $i \in I$, then the extended game $\Gamma^{*}=\left(I, F_{i}, \Omega_{i}\right)$ has a Nash equilibrium point.

PROOF. Under the assumptions that $d_{i i}>0, b_{i 0} \geq 0$ and $\partial_{i} \neq 0$ each of the functionals $J_{i}\left(u_{i}, \cdot\right), J_{i 1}\left(u_{i}, \cdot\right)$ and $J_{i 2}\left(u_{i}, \cdot\right)$ is strictly concave and continuous on the set $\left(\Omega_{i}, \tau_{i}\right)$ and thus $F_{i}$ is also strictly concave on $\left(\Omega_{i}, \tau_{i}\right)$. The set $u_{i}(u)=$ 
$\arg \max _{u_{i} \in \Omega_{i}} F_{i}\left(u_{i}, \cdot\right)$ is a singleton (and thus the multifunction $u_{i}(\cdot)$ is continuous on $\left.\left(\Omega_{i}, \tau_{i}\right)\right)$.

Consider the mapping $u(\cdot):(\Omega, \tau) \mapsto(\Omega, \tau)$

$$
u \mapsto\left(u_{1}(u), \ldots, u_{N}(u)\right)
$$

which is a continuous mapping as the composition and the Cartesian product of continuous mappings. The extended game $\Gamma^{*}$ has a Nash equilibrium $u^{*}$ if and only if $u^{*}$ is a fixed point of the mapping $u(\cdot)$. Since $(\Omega, \tau)$ is a convex and compact set, it follows from Tychonoff's fixed point theorem that $u(\cdot)$ has a fixed point $u^{*}$. This concludes the proof of the existence of a Nash point.

The extended game $\Gamma^{*}$ is a more general game than $\Gamma^{1}, \Gamma^{2}$ and $\Gamma$. In fact, each of the games $\Gamma^{1}, \Gamma^{2}$ and $\Gamma$ is a special case of $\Gamma^{*}$. Under the conditions contained in the theorem above a Nash equilibrium exists no matter whether a firm plays its Profit objective or its Capacity Management objective or just its Change of Technology objective. Each firm is therefore given three objective options, which may reflect management options in a technological service industry. Thus $\Gamma^{*}$ can be played in $3^{N}$ different ways.

\section{Conclusions}

The differential game that was considered by Kostreva [5] has been transformed into an equivalent infinite dimensional game. The existence of Nash equilibria has been proved for several variants of the new game. It has been shown in each case, under mild conditions on the target utilization coefficients of productive capacity $\partial_{i}$, the initial levels of per unit production cost which are reducible through the acquisition of new technology $\left(b_{i 0}\right)$ and the input constants for demand equations $\left(d_{i i}\right)$. These results have been made possible due to the transformations above. The methods used in this paper are also applicable to the model used by Gaimon [4], which corresponds to a single firm rather than a game, and that model lacks a proof of existence of an optimal solution.

The result of Polyak [7] has been used in its strong form since the class of strictly concave functions is a subset of the set of strictly quasi-concave functions on a given set. Thus the conditions of this paper may be made slightly more general, perhaps with some loss of economic motivation.

Following these transformations, a solution may be proved by means of fixed point theory. A more general treatment, covering large classes of games in many different contexts, is given in Abalo and Kostreva [1]. By means of this general plan of attack, many differential games may be solved, and we propose to extend the approach in future research. 


\section{References}

[1] K. Y. Abalo and M. M. Kostreva, "Fixed points, Nash games and their organizations", Top. Meth. Nonl. Analysis 8 (1997) 205-215.

[2] L. C. Cheng and E. Dinopoulos, "Schumpeterian growth and international business cycles", Amer. Econ. Rev. 82 (1992) 409-414.

[3] A. Friedman, Differential games (American Mathematical Society, Providence, RI, 1974).

[4] C. Gaimon, "The acquisition of new technology and its impact on dynamic pricing policies", in Studies in the Management Science and Systems (ed. B. Lev), Volume 13, (North-Holland, New York, 1986) 187-206.

[5] M. M. Kostreva, "Strategic analysis for a technological service industry: a differential game model", J. Oper. Res. Soc. 41 (1990) 573-582.

[6] N. Levinson, "A class of continuous linear programming problems", J. Math. Anal. Appl. 16 (1966) 73-83.

[7] B. T. Polyak, "Existence theorems and the convergence of minimizing sequences in extremum problems with restrictions", Sov. Math. 7 (1966) 72-75.

[8] F. Riesz and B. Sz.-Nagy, Leçons d'analyse fonctionnelle (Académie des Sciences de Hongrie, Budapest, Hungary, 1955).

[9] L. H. Roller and M. M. Tombak, "Competition and investment in flexible technologies", Man. Sci. 39 (1993) 107-114.

[10] M. M. Tombak, “A strategic analysis of flexible manufacturing systems", Euro. J. Oper. Res. 47 (1990) 225-238.

[11] A. Tychonoff, "Ein fixpunktsatz", Math. Arm. 111 (1935) 767-776.

[12] P. Varaiya, " $N$-person nonzero sum differential games with linear dynamics", SIAM J. Control 8 (1970) 441-449.

[13] M. Vidyasagar, "A new approach to $N$-person, nonzero-sum, linear differential games", J. Opt. Theory Appl. 18 (1976) 171-175. 